\title{
Special topic on aggregation-induced emission
}

\author{
Anjun Qin $^{1 *} \&$ Ben Zhong Tang ${ }^{1,2 *}$ \\ ${ }^{1}$ State Key Laboratory of Luminescent Materials and Devices, Center for Aggregation-Induced Emission, Guangzhou International Campus, \\ South China University of Technology, Guangzhou 510640, China; \\ 2 Department of Chemistry, Hong Kong Branch of National Engineering Research Center for Tissue Restoration and Reconstruction, Institute \\ for Advanced Study, and Department of Chemical and Biological Engineering, The Hong Kong University of Science \& Technology, \\ Hong Kong, China
}

Received July 20, 2018; accepted July 23, 2018; published online July 30, 2018

Citation: $\quad$ Qin AJ, Tang BZ. Special topic on aggregation-induced emission. Sci China Chem, 2018, 61: 879-881, https://doi.org/10.1007/s11426-018-9338-5

Aggregation-induced emission (AIE), conceptually termed in 2001 by our group at the Hong Kong University of Science \& Technology [1], refers to a unique phenomenon that a kind of nonemissive or weakly emissive molecules in their solution states become highly luminescent in their aggregate or solid states [2]. The AIE is exactly opposite to the general knowledge of aggregation-caused quenching (ACQ), which had been summarized by Birks in his classic book on Photophysics of Aromatic Molecules as "common (phenomenon) to most aromatic hydrocarbons and their derivatives" in 1970. Through a series of experimental investigation and theoretical calculation, restriction of intramolecular motion (RIM), which includes restriction of intramolecular rotation (RIR) and restriction of intramolecular vibration (RIV) has been rationalized as the main cause for the AIE effect [3].

AIE obeys the natural process of aggregation that is energy-favorable, avoids the complicated molecular design and physical approaches to inhibit aggregate formation, which finally gives rise to new models, new theories, new luminescent materials, new research fields, and new knowledge [2]. Owing to its theoretical importance and promising applications, AIE has aroused wide attention and enthusiastic participation of scientists from more than 80 countries and regions, including China, USA, Japan, Singapore, German, Italy, Australia, UK, and so on. Currently, there are hundreds of research groups working on

*Corresponding authors (email: msqinaj@scut.edu.cn; tangbenz@ust.hk)
AIE-related areas, and AIE has become one of the hot research topics in chemistry and materials fields. As a result, the research achievements have shown significant influence on and promoted the development of related scientific areas.

To highlight the fruitful outcome in the AIE area, several special issues/topics in prestigious international journals, such as ACS Applied Materials Interfaces in 2018, Materials Chemistry Frontiers in 2017, Faraday Discussion in 2017, and Small in 2016 have been published. We also edited special issues or topics in Chinese journals of Science China Chemistry in 2013 and Acta Sinica Chemica (in Chinese) in 2016. The contributors of the papers published in these two journals mostly come from China. To show the fruitful achievement on AIE from international scientists, we edit this special topic for Science China Chemistry. It is worth noting that the contributors are mostly international scientists, coming from Japan, Singapore, Germany, UK, Italy, Australia and Hong Kong, China. Moreover, the international collaborated results are also included.

The contents of this special topic cover the current hot researches including design and synthesis of new luminogens with AIE feature (AIEgens), applications of AIEgens in sensing, optoelectronic and biologic fields.

In the aspect of generation of new AIEgens, Shimizu and coworker from Japan designed and systematically studied a kind of 2,6-bis(diarylamino)benzo-phenone derivatives. The results showed that these fluorophores are also AIE-active and exhibit the delayed fluorescence at the same time.

Tsutsumi and coworkers from Japan designed and syn- 
thesized two thermally stable gold(I) NHC complexes, which exhibit crystallization-induced phosphorescence and aggregation-controlled emission.

Müller and coworkers from Germany synthesized a comprehensive substance library of 5-(hetero)aryl-thien-2yl substituted 3-ethynyl quinoxaline derivatives by Pd-catalyzed arylation and amination. Among the resultant 18 donor- $\pi$-acceptor fluorophores, two with 5-(hetero)aryl-thien2-yl substituted 3-ethynyl quinoxaline structures exhibited the unique AIE phenomenon.

In the AIE mechanism related area, Pucci and coworkers from Italy prepared new block copolymers consisting of a styrene block and a perfluorohexylethyl acrylate block that was endowed with fluorescence features by the covalent incorporation of a deliberately small amount of the 2-cyano-2-[4-vinyl(1,1'-biphenyl)-4'-yl]vinyljulolidine. The block copolymers were used to form the top-layer of two-layer films as vapochromic sensors of volatile organic compounds by utilizing the processes of restriction of intramolecular rotation, and twist intramolecular chargetransfer.

Chujo, Tanaka and coworkers from Japan compared the anchoring effect on electronic properties of the helicene-like bibenzothiophene between $o$-carborane and 5,6-dicarbanido-decaborane. The results indicated that the $\pi$-conjugation on the distorted structure supported by the nido-decaborane anchor played a significant role in suppressing aggregation-caused quenching followed by presenting solid-state emission with stimuli responsiveness.

In the sensing field based on AIE probe, Tang, Hao and coworkers from Hong Kong and Mainland, China described a general powder dusting method for latent fingerprint using the TPE-based AIEgens of TPE-DPA, TPE-2DPA and TPE-4DPA. After doping with magnetic powders, the system could be applied for visualization of latent fingerprint on various smooth and porous substrates, including glass, stainless steel, leaf, ceram, plastic bag, lime wall, wood and paper money with high resolution.

In the area of opto-electronic application, Scherf and coworkers from Germany and UK synthesized mono- and tri-TPE-substituted BODIPY derivatives, which show decreased emission and AIE feature due to the TICT and RIR processes in low and high water fraction in tetrahydrofuran (THF)/water mixtures, respectively. Interestingly, when blending the TPE-substituted BODIPY fluorophores into a polymer matrix of poly[(9,9-di- $n$-octylfluorene-2,7-diyl)alt-(benzo[2,1,3]thiadiazol-4,7-diyl)] (F8BT), enhanced emission was observed, which has been successfully used to fabricated OLEDs that exhibit electroluminescence (EL) peaked in the range of $650-700 \mathrm{~nm}$, with up to $1.8 \%$ external quantum efficiency (EQE) and $2 \mathrm{~mW} / \mathrm{cm}^{2}$ radiance.

In the aspect of bio-related applications, Hong and coworkers from Australia briefly reviewed recent progress in the development of biothiol-specific AIEgens, especially focusing on the molecular design principles to target different types of biothiols and the corresponding sensing mechanisms. Moreover, the potential of future design and development of multi-functional bioprobes was also discussed.

Tang YH from Australia, Tang BZ from Hong Kong China and their collaborators from Mainland China utilized multiplexed imaging on HeLa cells stained with a combination of nuclear dye Hoechst 33258, mitochondria, lysosome and cytoplasmic protein specific AIEgens of TPE-Ph-In, 2M-DABS and BSPOTPE to successfully investigate the intracellular changes during early and middle apoptosis.

Liu from Singapore, Hua from Mainland China together with their coworkers reported the design and synthesis of three NIR emissive glyco-probes based on diketopyrrolopyrrole (DPPs) conjugated with two (DPPG), four (DPPF-G) and six (DPPS-G) galactose groups. All three molecules could probe lectins with excellent sensitivity and selectivity based on the AIE mechanism.

The above works have shed light on the bright future of AIE research. We are confident that great progress will be further made with continue efforts devoted by researchers worldwide, and more and more innovative and original results will be presented. These achievements will in turn make great contributions to the science \& technology development.

We would like to thank all the contributors and reviewers for their time and efforts, and thank Dr. Jianfen Zheng and other editors from the editorial office of Science China Chemistry for their professional support.

1 Luo J, Xie Z, Lam JWY, Cheng L, Tang BZ, Chen H, Qiu C, Kwok HS, Zhan X, Liu Y, Zhu D. Chem Commun, 2001: 1740-1741

2 Mei J, Leung NLC, Kwok RTK, Lam JWY, Tang BZ. Chem Rev, 2015, 115: 11718-11940

3 Mei J, Hong Y, Lam JWY, Qin A, Tang Y, Tang BZ. Adv Mater, 2014, 26: 5429-5479 


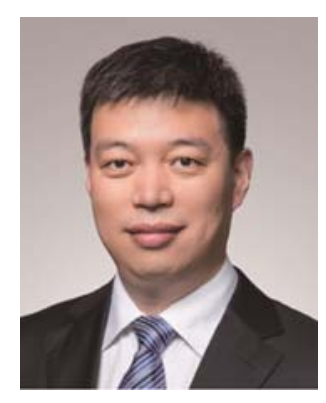

Prof. Anjun Qin received his BSc and PhD degrees from Shanxi University and Institute of Chemistry of the Chinese Academy of Sciences in 1999 and 2004, respectively. He conducted his postdoctoral research at the Hong Kong University of Science \& Technology (HKUST) and Zhejiang University (ZJU) under the supervision of Prof. Ben Zhong Tang during 2005-2008. He joined ZJU as an Associate Professor at the end of 2008 and moved to South China University of Technology with promotion to Full Professor in 2013. His current research interests include the development of new polymerizations based on triple-bond building blocks, and the construction of organic/polymeric functional materials.

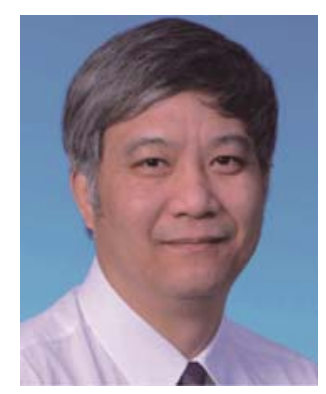

Prof. Ben Zhong Tang received his PhD degree from Kyoto University in 1988. He conducted his postdoctoral work at University of Toronto in 1989-1994. He joined HKUST in 1994 and was promoted to Chair Professor in 2008 and Stephen K. C. Cheong Professor of Science in 2013. He was elected to the Chinese Academy of Sciences in 2009. His research interests include the exploration of new polymerization based on triple-bond building blocks and investigation of aggregation-induced emission. 